3 Benumof JL. The American Society of Anesthesiologists' management of the difficult airway algorithm and explanation-analysis of the algorithm. In: Benumof JL (Ed). Airway Management. Principle and Practice. St. Louis, Missouri: Mosby, 1996: 143-56.

\section{Cardiovascular responses to endotra- cheal intubation with the Bullard and the Macintosh laryngoscopes}

To the Editor:

Since the Bullard laryngoscope (BL) is anatomically shaped, it is considered less invasive than the Macintosh laryngoscope (ML) is. We compared cardiovascular changes following endotracheal intubation and times required for intubation between the two laryngoscopes.

After obtaining Institutional approval and informed consent, 30 patients without hypertension were studied. Endotracheal intubation was performed by BL (15 patients) or ML (15 patients), following induction of general anesthesia. Systolic and diastolic blood pressure (SBP and DBP), heart rate just before and one, two, three, four and five minutes after intubation were measured. The time required to visualize the glottis (T1), to place the tube (T2), and to complete intubation (T3) were recorded. All intubations were successful at the first attempt. The increase in SBP displayed a tendency to be smaller in the Bullard group than in the Macintosh group (12 $\mathrm{mmHg}$ difference, two minutes after intubation), but the difference was not statistically significant (Figure). There were no differences in DBP and in heart rate between the groups. Time to visualize the vocal cords, time required to place the endotracheal tube and time to complete intubation were significantly longer with the $\operatorname{BL}(7.7,17.5$ and $21.3 \mathrm{sec}$, respectively) than with the $\operatorname{ML}(5.3,12.7$ and $14.7 \mathrm{sec}$, respectively; $P<0.05)$.

Although the time to complete intubation with the BL was statistically longer, the $6.6 \mathrm{sec}$ difference does not seem clinically important. Although we were unable to demonstrate that the $\mathrm{BL}$ is less invasive in patients without hypertension, further studies (larger sample size; hypertensive patients) appear warranted.

\section{Kazukuni Araki MD}

Ryoichi Nomura MD

Naohisa Tsuchiya MD

Yokiko Yoshikawa MD

Ohtsu, Japan

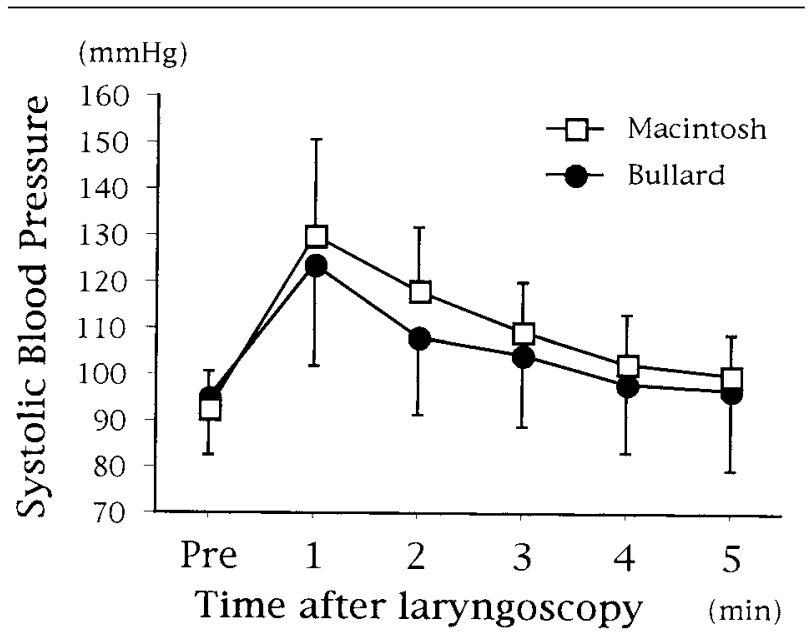

FIGURE Comparison of systolic blood pressure changes between the two laryngoscopes. Data are mean \pm SD. The difference was not significant between the groups. $P=0.051$ by repeated measure ANOVA. Pre $=$ before intubation .

\section{Positioning the double-lumen endo- bronchial tube}

\section{To the Editor:}

We read with interest an article by Fortier et al. ${ }^{1}$ regarding new landmarks to improve the positioning of the left Broncho-Cath ${ }^{\mathrm{TM}}$ double-lumen tube (DLT). Through the bronchial tube and by transparency across the wall, the position of the DLT was adjusted so that the carina is midway between the black radiopaque line and the top of the bronchial cuff. ${ }^{1}$ Through the bronchial lumen, however, the DLT appears to be located deeper relative to the black radiopaque line because the line of vision through the fibreoptic bronchoscope is almost vertical. Thus, the bronchial cuff must have been placed more proximally than intended by the authors. Figure 3 of the articlel may be self-explanatory.

When a DLT is located within the margin of safety, defined as the difference between the length of the main bronchus and the length of the tube between the top of the bronchial cuff and the tip, ${ }^{2}$ the DLT can be moved over the difference and still be correctly positioned. When the carina is at the level midway between the top of the bronchial cuff and the black radiopaque line, the tube length below the carina is about $35 \mathrm{~mm}$, because the length of the bronchial tube of the DLT is $40 \mathrm{~mm} .^{3}$ The possibility of the bronchial tube tip not being within the acceptable 